\title{
Hamiltonian strongly regular graphs
}

\author{
A. E. Brouwer \& W. H. Haemers
}

2008-03-04

\begin{abstract}
We give a sufficient condition for a distance-regular graph to be Hamiltonian. In particular, the Petersen graph is the only connected nonHamiltonian strongly regular graph on fewer than 99 vertices.
\end{abstract}

\section{Introduction}

For a graph $\Gamma$, let $\alpha(\Gamma)$ denote the independence number (the maximum size of an independent set) and let $\kappa(\Gamma)$ denote the vertex connectivity (the minimum number of vertices one has to remove from $\Gamma$ in order to disconnect it; note that the graph without vertices is not connected).

Chvátal \& Erdős [5] proved that every graph $\Gamma$ on at least three vertices, with $\alpha(\Gamma) \leq \kappa(\Gamma)$ is Hamiltonian. Hoffman showed (cf. [1], 1.3.2) that every regular graph $\Gamma$ on $n$ vertices with valency $k(k>0)$ and smallest eigenvalue $s$ satisfies $\alpha(\Gamma) \leq-n s /(k-s)$. It follows that every $k$-regular $k$-connected graph on $n>2$ vertices with $-n s /(k-s)<k+1$ is Hamiltonian.

However, for many interesting $k$-regular $k$-connected graphs we have $-n s /(k-s)=k+1$. This includes the Petersen graph (which has $(n, k, s)=(10,3,-2))$. Here we show:

Theorem 1 Let $\Gamma$ be a k-regular $k$-connected graph with $n$ vertices and smallest eigenvalue $s$ which is not the Petersen graph. If $k>1$ and $-n s \leq(k+1)(k-s)$ then $\Gamma$ is Hamiltonian.

Brouwer \& Koolen [3] proved that every non-complete distance-regular graph of degree $k$ is $k$-connected. Hence

Corollary 2 Let $\Gamma$ be a $k$-regular distance-regular graph with $n$ vertices and smallest eigenvalue $s$ which is not the Petersen graph. If $k>1$ and $-n s \leq(k+1)(k-s)$ then $\Gamma$ is Hamiltonian.

Several distance-regular graphs satisfy this inequality (and some with equality). For connected strongly regular graphs (i.e., distance regular graphs of diameter 2), this becomes

Corollary 3 Let $\Gamma$ be a connected strongly regular graph with parameters $(n, k, \lambda, \mu)$ and smallest eigenvalue $s$. If $s$ is not integral, or if $-s \leq \mu+1$, then $\Gamma$ is Hamiltonian.

(Here $\mu$ is the number of common neighbors of two nonadjacent vertices.) This corollary covers most admissible parameter sets for strongly regular graphs. We conjecture that the Petersen graph is the only connected strongly regular graph that is not Hamiltonian.

An important ingredient of the proof of the theorem is the following characterization of the case of equality in Hoffman's bound (cf. [1], 1.3.2). 
Lemma 4 Let $\Gamma$ be a $k$-regular graph $(k>0)$ with $n$ vertices and smallest eigenvalue s. Then $\alpha(\Gamma) \leq-n s /(k-s)$. A coclique $S$ satisfies the bound with equality, if and only if every vertex not in $S$ is adjacent to exactly $-s$ vertices of $S$.

\section{Rearranging cycles}

Suppose the graph $\Gamma$ is connected and non-Hamiltonian, with at least one cycle. Let $C$ be a longest cycle. Provide $C$ with a cyclic order, and write $x^{+}$(resp. $x^{-}$) for the successor (predecessor) of $x$ on $C$. Write $D^{+}$(resp. $D^{-}$) for $\left\{u^{+} \mid u \in D\right\}$ (resp. $\left\{u^{-} \mid u \in D\right\}$ ) for any subset $D$ of $C$. When describing subpaths of $C$, use $u \ldots v$ for the path from $u$ to $v$ in the cyclic order of $C$, and $u,,, v$ for the path from $u$ to $v$ in the opposite direction. For a set of vertices $X$, let $N(X)$ be the set of vertices not in $X$ but with a neighbor in $X$. Let $H$ be a component of $\Gamma \backslash C$. Let $K=N(H)$. Then $K \subseteq C$.

(i) If $u, v \in K$ and $v=u^{+}$, then $u H v \ldots u$ is a longer cycle, if $u H v$ denotes a path joining $u$ and $v$ via vertices (at least one) in $H$. Contradiction. So, $K \cap K^{-}=K \cap K^{+}=\emptyset$ and $K$ is a vertex cut of $\Gamma$ and $|K|=\left|K^{+}\right|=\left|K^{-}\right| \geq \kappa(\Gamma)$.

(ii) If $u, v \in K$ and $u^{+}$is adjacent to $v^{+}$, then $u H v,,, u^{+} v^{+} \ldots u$ is a longer cycle, contradiction. So $K^{+}$(and $K^{-}$) are cocliques nonadjacent to any point of $H$, and $\alpha(\Gamma) \geq|K|+\alpha(H)$. This argument proves the Chvátal-Erdős theorem.

Now assume that $\alpha(\Gamma)=\kappa(\Gamma)+1$. Then $\kappa(\Gamma)=|K|$ and $\alpha(H)=1$, so $H$ is a clique. Assume moreover that whenever $S$ is a coclique of size $\alpha(\Gamma)$, each vertex outside $S$ has at least two neighbors in $S$.

Claim: $|C|=n-1$.

Proof. Suppose $h, h^{\prime} \notin C$, with $h \in H$, and consider the coclique $S=K^{+} \cup\{h\}$ of size $\alpha(\Gamma)$. Since $h^{\prime} \notin S$ the vertex $h^{\prime}$ has at least two neighbours in $S$. Since $K \cap K^{+}=\emptyset$, it follows that $h^{\prime}$ belongs to a component $H^{\prime} \neq H$ of $\Gamma \backslash C$ and has two neighbors $u^{+}$and $v^{+}$in $K^{+}$. But now $u h v,,, u^{+} h^{\prime} v^{+} \ldots u$ is a longer cycle. Contradiction.

Assume that $\Gamma$ is not a complete bipartite graph $K_{t, t+1}$. Put $H=\{h\}$.

Claim: $K^{+} \cap K^{-}=\emptyset$.

Proof. Suppose $a=u^{+}=v^{-}$with $u, v \in K$. Then $C^{\prime}=h v \ldots u h$ is another maximum cycle, missing $a$. Let $A$ be the set of neighbors of $a$. Assume there exists a $w \in K \backslash A$. The set $S=A^{-} \cup\{h\}$ is a coclique of size $\alpha(\Gamma)$, and $w^{-} \notin S$, and $w^{-} \notin K$, so $w^{-}$has at least one neighbor $b$ in $A^{-} \backslash\{a\}$. If $b$ lies between $a$ and $w$, then $h w \ldots a b^{+} \ldots w^{-} b,,, v h$ is a longer cycle. If $b$ lies between $w$ and $a$, then $h w \ldots b w^{-},,, a b^{+} \ldots u h$ is a longer cycle. So there is no such vertex $w$, and hence $K=A$.

If $K^{+}=K^{-}$then $\kappa(\Gamma)=(n-1) / 2$. Since each vertex has at least $\kappa(\Gamma)$ neighbours, $\Gamma$ is $K_{t, t+1}$, which was excluded.

Hence we may assume that $v$ was chosen such that $v^{+} \in K^{+} \backslash K^{-}$. Let $w$ be the first vertex in $C$ after $v$ such that $w^{-} \in K^{-} \backslash K^{+}$. Now $w^{-}$has at least two neighbors in $K^{+} \cup\{h\}$, so certainly one neighbor $x^{+} \in K^{+}$is different from $v^{+}$and hence lies between $w$ and $a$. But now $h w \ldots x a,,, x^{+} w^{-},,, v h$ is a longer cycle, contradiction.

Now assume that whenever $S$ is a coclique of size $\alpha(\Gamma)$, each vertex outside $S$ has at least three neighbors in $S$. 
Call an edge $\{a, b\} \in E(\Gamma)$ a special cord of $C$ if $a, b \in C,\{a, b\} \notin E(C)$, and $a$ or $b$ lies in $K^{+} \cup K^{-}$. The length of a special cord $\{a, b\}$ is the length of the shortest (undirected) path between $a$ and $b$ via $C$. Let $\{a, b\}$ be a special cord of minimum length for a cycle $C$, where the minimum is taken over all possible special cords and maximum cycles. Assume that $C$ is oriented from $a$ to $b$.

If $a^{+} \notin K^{+}$then there is a vertex $u^{+} \in K^{+} \backslash\left\{a, a^{++}\right\}$, adjacent to $a^{+}$. Since $\{a, b\}$ is a minimal special cord, $u^{+}$is not in $a \ldots b$. Similarly, if $b^{-} \notin K^{+}$there is a vertex $v^{+} \in K^{+} \backslash\left\{b^{--}, b\right\}$ adjacent to $b^{-}$, but not in $a \ldots b$. Now $h u,,, v^{+} b^{-},,, a^{+} u^{+} \ldots a b \ldots v h$ or $h v,,, u^{+} a^{+} \ldots b^{-} v^{+} \ldots a b \ldots u h$ is a longer cycle.

In a similar way we find a longer cycle if $a^{+} \notin K^{-}$and $b^{-} \notin K^{-}$. It is impossible that $a^{+} \in K^{+}$and $b^{-} \in K^{-}$because $K \cap\left(K^{+} \cup K^{-}\right)=\emptyset$. Therefore $a^{+} \in K^{-}, b^{-} \in K^{+}$and $a \in K^{+}$or $b \in K^{-}$. By symmetry, we may assume $a \in K^{+}$.

Now $C^{\prime}=h a^{-},, b a \ldots b^{--} h$ is another maximum cycle, missing $b^{-}$. If $a^{++} \neq b^{--}$, then $C^{\prime}$ has a special cord $\left\{a^{++}, h\right\}$, which is shorter than the special cord $\{a, b\}$ of $C$, contradiction. So $a^{++}=b^{--}$. (Note that the Petersen graph has this structure.) Let $E$ be the set of neighbors of $b^{-}$ distinct from $b, b^{--}$. Then $S=E^{-} \cup\left\{b^{-}, a, h\right\}$ is a coclique of size $\alpha(\Gamma)$.

We have $a^{+} \in K^{-}$, so $a^{+}$has a neighbor $u^{+}$in $K^{+} \backslash\{a\}$. Now $C^{\prime \prime}=h a^{-},,, u^{+} a^{+} a b \ldots u h$ is a circuit of length $n-2$ missing $b^{--}$and $b^{-}$.

Since $b^{--} \notin S$, it follows that $b^{--}$has a neighbor $c^{-} \in E^{-}$. Now $P=c b^{-} b^{--} c^{-}$is a path of length 3. Since $b^{-} \in K^{+}$we have $c \neq u^{+}$so $\left\{c^{-}, c\right\}$ is an edge of $C^{\prime \prime}$. Replacing this edge by $P$ gives a Hamiltonian circuit. This proves:

Proposition 5 Suppose that $\alpha(\Gamma) \leq \kappa(\Gamma)+1$, and that each vertex outside any coclique $S$ of size $\alpha(\Gamma)$ has at least 3 neighbours in $S$. Then $\Gamma$ is Hamiltonian, or $\Gamma$ is $K_{t, t+1}$ (with $t \geq 2$ ).

Corollary 6 Let $\Gamma$ be a $k$-regular and $k$-connected graph with smallest eigenvalue $s<-2$. If $-n s /(k-s) \leq k+1$, then $\Gamma$ is Hamiltonian.

\section{Smallest eigenvalue -2}

Suppose $\Gamma$ is $k$-regular with smallest eigenvalue -2 . Then $\Gamma$ is a line graph or belongs to a finite list of 187 so called regular exceptional graphs (see [4] or [6]). These graphs and their spectra are known ([6], Table A3). For these graphs Hoffman's coclique bound equals $2 n /(k+2)$, and for only five cases this number is equal to $k+1$. All five have $n=10$ and $k=3$ (see [6], p. 218 for the drawings of these five graphs). One is the Petersen graph, but the other four are easily seen to be Hamiltonian. So we can conclude:

Proposition 7 The Petersen graph is the only non-Hamiltonian k-regular exceptional graph with a coclique of size $k+1$.

Next we deal with the case that $\Gamma$ is a $k$-regular line graph.

Proposition 8 A $k$-connected $k$-regular line graph $\Gamma$ on $n(n \geq 3)$ vertices satisfying $\alpha(\Gamma)=2 n /(k+2) \leq k+1$, is Hamiltonian.

Proof. Let $\Gamma$ be the line graph of $\Gamma^{\prime}$ with $n^{\prime}$ vertices. Note that a coclique in $\Gamma$ corresponds to a matching in $\Gamma^{\prime}$. It is well-known that $\Gamma^{\prime}$ is either regular or bipartite biregular. Assume $\Gamma^{\prime}$ is bipartite biregular. Let $n_{1}^{\prime}$ 
and $n_{2}^{\prime}\left(n_{1}^{\prime} \leq n_{2}^{\prime}\right)$ be the sizes of the two parts with degrees $k_{1}^{\prime}$ and $k_{2}^{\prime}$, respectively. Then $k=k_{1}^{\prime}+k_{2}^{\prime}-2, n=n_{1}^{\prime} k_{1}^{\prime}=n_{2}^{\prime} k_{2}^{\prime}$, and $k_{1}^{\prime} \geq k_{2}^{\prime}$. Clearly, a matching in $\Gamma^{\prime}$ has at most $n_{1}^{\prime}$ edges, so $\alpha(\Gamma) \leq n_{1}^{\prime}$. On the other hand, $2 n /(k+2)=2 n_{1}^{\prime} k_{1}^{\prime} /\left(k_{1}^{\prime}+k_{2}^{\prime}\right) \geq n_{1}^{\prime}$ with equality only if $k_{1}^{\prime}=k_{2}^{\prime}$. This shows that $\Gamma^{\prime}$ is regular (of degree $k^{\prime}$ say).

A sequence $\left(e_{1}, \ldots, e_{\ell}\right)$ of $\ell$ distinct edges of a graph is called sequential if $e_{i}$ and $e_{i+1}$ intersect for $i=1, \ldots, \ell-1$. A graph is called sequential if it admits a sequential sequence $\left(e_{1}, \ldots, e_{m}\right)$ consisting of all edges, such that $e_{1}$ intersects $e_{m}$. It is clear that a graph is sequential if and only if its line graph is Hamiltonian. We will show that $\Gamma^{\prime}$ is sequential. If $k^{\prime}$ is even, then $\Gamma^{\prime}$ is Eulerian and therefore sequential. So assume that $k^{\prime}$ is odd. Note that $n=n^{\prime} k^{\prime} / 2$ and $k=2\left(k^{\prime}-1\right)$. Since $\alpha(\Gamma)=2 n /(k+2)=n^{\prime} / 2$, $\Gamma^{\prime}$ has a perfect matching $M$ (say). Consider the graph $\Gamma^{\prime \prime}$, obtained from $\Gamma^{\prime}$ by deleting $M$. If $\Gamma^{\prime \prime}$ is connected, $\Gamma^{\prime \prime}$ is Eulerian, and $\Gamma^{\prime}$ is easily seen to be sequential. If $\Gamma^{\prime \prime}$ is not connected, it consists of two disjoint $\left(k^{\prime}-1\right)$ regular components, both with $n^{\prime} / 2$ vertices, and all edges of $M$ meet both components. (Indeed, since $\Gamma$ is $k$-connected, and $n^{\prime} / 2=\alpha(\Gamma) \leq k+1$, $\Gamma^{\prime}$ cannot be separated by removing fewer than $n^{\prime} / 2$ edges.) The two components $\Gamma_{1}$ and $\Gamma_{2}$ of $\Gamma^{\prime \prime}$ are Eulerian. Consider two edges $\{w, x\}$ and $\{y, z\}$ from $M$ such that $\{x, y\}$ is an edge in $\Gamma_{2}$. From an Eulerian cycle of $\Gamma_{2}$ one easily constructs a sequential sequence $S$ of $\Gamma^{\prime}$, such that $S$ contains all edges of $\Gamma_{2}$ and $M$, starts with $\{w, x\}$ and ends with $\{y, z\}$. If $\{w, z\}$ is an edge of $\Gamma_{1}$ we can insert $S$ in an Eulerian tour in $\Gamma_{1}$ right before or after $\{w, z\}$ and find that $\Gamma^{\prime}$ is sequential. If $w$ and $z$ are not adjacent, they have a common neighbor $v$ in $\Gamma_{1}$ (since $\left.2 k^{\prime}>n^{\prime} / 2\right)$. Now consider the graph obtained from $\Gamma_{2}$ by deleting the edges $\{v, w\}$ and $\{v, z\}$ and adding the edges $\{w, x\}$ and $\{x, y\}$ and $\{y, z\}$. Now we can insert $S$ in an Eulerian tour of this latter graph, minus the three added edges, and also insert the two removed edges again, and find that $\Gamma^{\prime}$ is sequential.

The two propositions above together with the theorem of Chvátal and Erdös prove the main theorem if $s=-2$. If $s=-1$, then $\Gamma=K_{n}$, and the result is trivial. With Corollary 6, this completes the proof of the main theorem.

\section{Examples}

Several distance-regular graphs of small diameter satisfy the inequality of Corollary 2. An interesting infinite family consists of the collinearity graphs of generalized quadrangles $G Q(r, t)$ from which a spread has been deleted (see [1], p. 385). These graphs have diameter 3 and $(n, k, s)=$ $((r+1)(r t+1), r t,-t)$. They satisfy the condition (with equality), and hence are Hamiltonian. The smallest of these has $r=t=2$, and is isomorphic to the line graph of the Petersen graph.

The distance-regular graphs with diameter 2 are precisely the connected strongly regular graphs. For these graphs the connectivity was determined already in [2]. Only few parameter sets for connected strongly regular graphs have $-s>\mu+1$. For $n \leq 100$ there are only five cases: 


\begin{tabular}{llcll}
$\#$ & $(v, k, \lambda, \mu)$ & $s$ & name & Hamiltonian \\
\hline 1 & $(50,7,0,1)$ & -3 & Hoffman-Singleton graph & yes \\
2 & $(56,10,0,2)$ & -4 & Gewirtz graph & yes \\
3 & $(77,16,0,4)$ & -6 & $M_{22}$ graph & yes \\
4 & $(99,14,1,2)$ & -4 & & $?$ \\
5 & $(100,22,0,6)$ & -8 & Higman-Sims graph & yes
\end{tabular}

In cases $1,2,3$ and 5 there is a unique strongly regular graph with the given parameters, and the graph is Hamiltonian by direct verification. (In case 5 Hamiltonicity also follows from the fact that the smallest eigenvalue has multiplicity 22 , so that $\alpha \leq 22$.)

In case 4, existence of a strongly regular graph is open. Thus, all connected strongly regular graphs on fewer than 99 vertices are Hamiltonian.

We conjecture that the Petersen graph is the only connected nonHamiltonian strongly regular graph.

\section{References}

[1] A. E. Brouwer, A. M. Cohen \& A. Neumaier, Distance-Regular Graphs, Springer-Verlag, 1989.

[2] A. E. Brouwer \& D. M. Mesner, The connectivity of strongly regular graphs, Europ. J. Combin. 6 (1985) 215-216.

[3] A. E. Brouwer \& J. H. Koolen, The vertex-connectivity of a distanceregular graph, Europ. J. Combin. (to appear).

[4] F. C. Bussemaker, D. Cvetković \& J. J. Seidel, Graphs related to exceptional root systems, T. H. Report 76-WSK-05, Technical University Eindhoven, 1976.

[5] V. Chvátal \& P. Erdős, A note on Hamiltonian circuits, Discr. Math. 2 (1972) 111-113.

[6] D. Cvetković, P. Rowlinson \& S. Simić, Spectral Generalizations of Line Graphs: On graphs with least eigenvalue -2, Cambridge Univ. Press, 2004. 